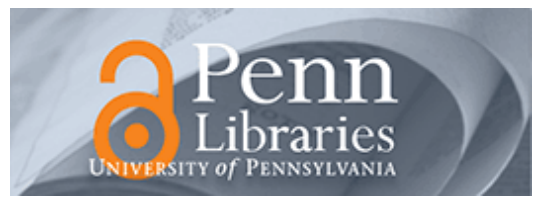

University of Pennsylvania

ScholarlyCommons

$10-9-2007$

\title{
Novel Defect Structures in Nematic Liquid Crystal Shells
}

\author{
Alberto Fernández-Nieves \\ Vincenzo Vitelli \\ University of Pennsylvania \\ Andrew S. Utada \\ Harvard University \\ Darren R. Link \\ Harvard University \\ Manuel Márquez \\ Arizona State University
}

See next page for additional authors

Follow this and additional works at: https://repository.upenn.edu/physics_papers

Part of the Physics Commons

\section{Recommended Citation}

Fernández-Nieves, A., Vitelli, V., Utada, A. S., Link, D. R., Márquez, M., Nelson, D. R., \& Weitz, D. A. (2007). Novel Defect Structures in Nematic Liquid Crystal Shells. Retrieved from https://repository.upenn.edu/ physics_papers/134

\section{Suggested Citation:}

Fernábdez-Nieves, A., et al.. (2007). Novel Defect Structures in Nematic Liquid Crystal Shells. Physical Review Letters. 99, 157801.

(c) 2007 The American Physical Society

http://dx.doi.org/10.1103/PhysRevLett.99.157801

This paper is posted at ScholarlyCommons. https://repository.upenn.edu/physics_papers/134

For more information, please contact repository@pobox.upenn.edu. 


\title{
Novel Defect Structures in Nematic Liquid Crystal Shells
}

\author{
Abstract \\ We use double-emulsion drops to experimentally investigate the defect structures of spherical shells of \\ nematic liquid crystals. We uncover a rich scenario of coexisting defect structures dictated by the \\ unavoidable finite thickness of even the thinnest shell and by the thickness variation around the sphere. \\ These structures are characterized by a varying number of disclination lines and pairs of surface point \\ defects on the inner and outer surfaces of the nematic shell. In the limit of very thick shells the defect \\ structure ultimately merges with that of a bulk nematic liquid crystal drop. \\ Disciplines \\ Physical Sciences and Mathematics | Physics

\section{Comments} \\ Suggested Citation: \\ Fernábdez-Nieves, A., et al.. (2007). Novel Defect Structures in Nematic Liquid Crystal Shells. Physical \\ Review Letters. 99, 157801. \\ (C) 2007 The American Physical Society \\ http://dx.doi.org/10.1103/PhysRevLett.99.157801

\section{Author(s)} \\ Alberto Fernández-Nieves, Vincenzo Vitelli, Andrew S. Utada, Darren R. Link, Manuel Márquez, David R. \\ Nelson, and David A. Weitz
}




\title{
Novel Defect Structures in Nematic Liquid Crystal Shells
}

\author{
A. Fernández-Nieves, ${ }^{1,2}$ V. Vitelli, ${ }^{1,3}$ A. S. Utada, ${ }^{1}$ D. R. Link, ${ }^{1}$ M. Márquez, ${ }^{2,4}$ D. R. Nelson, ${ }^{1}$ and D. A. Weitz ${ }^{1}$ \\ ${ }^{1}$ Department of Physics and HSEAS, Harvard University, Cambridge, Massachusetts 02138, USA \\ ${ }^{2}$ Interdisciplinary Network of Emerging Science and Technology (INEST) Group, Research Center, Phillip Morris USA, \\ Richmond, Virginia 23298, USA \\ ${ }^{3}$ Department of Physics, University of Pennsylvania, Philadelphia, Pennsylvania 19104, USA \\ ${ }^{4}$ Harrington Department of Bioengineering, Arizona State University, Tempe, Arizona 85287, USA
}

(Received 29 June 2007; published 9 October 2007)

\begin{abstract}
We use double-emulsion drops to experimentally investigate the defect structures of spherical shells of nematic liquid crystals. We uncover a rich scenario of coexisting defect structures dictated by the unavoidable finite thickness of even the thinnest shell and by the thickness variation around the sphere. These structures are characterized by a varying number of disclination lines and pairs of surface point defects on the inner and outer surfaces of the nematic shell. In the limit of very thick shells the defect structure ultimately merges with that of a bulk nematic liquid crystal drop.
\end{abstract}

DOI: 10.1103/PhysRevLett.99.157801

PACS numbers: 61.30.Jf, 47.55.D-

Emulsions of liquid crystal in a continuous fluid such as water have technologically important uses. They also produce fascinating defect structures due to the confinement of the liquid crystal and the strong effect of the boundary conditions on the ordering at the interface. For example, nematic liquid crystals, constrained to lie parallel to the interface of a drop, possess two intrinsic defects, called boojums [1,2]. These defects are the inevitable consequence of the elastic energy of the liquid crystal in the drop and the parallel boundary conditions at the interface. Even more fascinating structures result when small water droplets are confined within the larger liquid crystal drop. The liquid-crystal-mediated interparticle interaction causes the small drops to align into chains [3,4]. The simplest case of this is when there is a single inner drop, leaving a shell of liquid crystal; in this case, the behavior should depend on the relative size of the inner drop and on the boundary conditions. For parallel boundary conditions, a small inner drop does not have a large effect; the boojum structure persists, but with two additional defects on the inner surface, as required by the surface boundary conditions [5]. However, as the size of the inner drop increases, the very nature of the defect structure evolves. For very thin shells, a totally new structure is predicted; instead of two defects, there are four defects at each surface, organized in a tetrahedral fashion [6,7]. However, despite the rich range of behavior expected, there have been no experimental investigations of defects in shells of nematic liquid crystal as the shell is thinned.

In this Letter, we investigate the behavior of liquid crystal shells as the their thickness is varied. For the thickest shells, we observe a defect structure similar to the boojum structure of a nematic drop. As the shell becomes thinner, there is a transition to the tetrahedral structure expected for very thin shells. However, for these thin shells, we also see two unexpected and new defect structures, consisting of two or three defects. These are the first observations of the rich behavior exhibited by shells of nematic liquid crystal.

To make spherical shells of liquid crystal we generate double emulsions using an axisymmetric microfluidic device [8]. Both the inner and outer fluids are water that contain $1 \mathrm{wt} \%$ poly-vinyl-alcohol (PVA), which stabilizes the double emulsion and enforces tangential boundary conditions for the nematic liquid crystal. We approximately match the density of the inner water to that of the liquid crystal, pentyl-cyano-biphenyl (5CB), by adding $\mathrm{D}_{2} \mathrm{O}$ to obtain $\rho_{5 \mathrm{CB}}-\rho_{\text {inner }}=2 \mathrm{~kg} / \mathrm{m}^{3}$. The resultant double-emulsion drops consist of an inner droplet of radius $a$, encapsulated within a shell of liquid crystal of outer radius $R$, all immersed in an aqueous solution. By varying the fluid flow rates, we can vary the thickness and size of the shells.

A typical example of a thick nematic shell is shown in Fig. 1, where, using bright field microscopy, we focus on the top [Fig. 1(a)], middle [Fig. 1(b)], and bottom [Fig. 1(c)] of the large drop; the smaller inner drop is located towards one edge as highlighted in Fig. 1(c). By viewing these same images through crossed polarizers we identify one boojum at the top of the large drop, as shown in Fig. 1(d), which corresponds to the same position as Fig. 1(a). There is a second boojum at the top of the smaller drop, as shown in Fig. 1(e) and in the magnified view, Fig. 1(h); this corresponds to the same position as Fig. 1(b). Topological constraints require a second boojum on both the inner and the outer surfaces. These can be observed in Fig. 1(f) which corresponds to the same position as Fig. 1(c). However, since the thickness of the shell is so small in this location we are unable to distinguish the two boojums and instead only observe a single one, even in the magnified image shown in Fig. 1(i). Nevertheless a second defect is required to satisfy the topological constraints, which are governed by the Poincaré theorem [9]. The resultant structure is reminiscent of a bipolar drop, where 

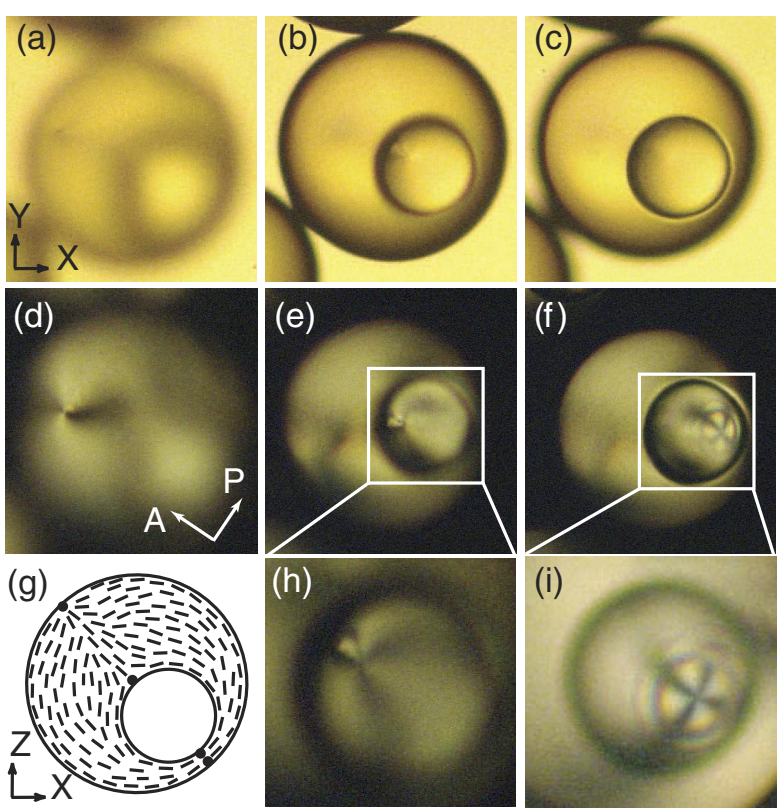

FIG. 1 (color online). Bright field (a)-(c) and cross-polarized (d) -(f) images of a density matched nematic shell. The inner fluid consists of a solution of $\mathrm{H}_{2} \mathrm{O}$ and $\mathrm{D}_{2} \mathrm{O}+$ PVA ( $1 \mathrm{wt} \%$ ), the middle fluid is the liquid crystal, and the outer fluid is $\mathrm{H}_{2} \mathrm{O}+$ PVA (1 wt \%). Drop sizes are $2 a=93 \mu \mathrm{m}$ and $2 R=206 \mu \mathrm{m}$. As the microscope focal plane is lowered, the upper (a), (d), intermediate (b),(e), and lower boojums (c),(f) can be identified. (g) Schematic of the director field configuration. (h), (i) are magnifications of the inner drop in (e), (f) after some rotation; this allows better visualization of the defects. The lower pair of boojums cannot be resolved with optical microscopy and thus appears as one.

the inner droplet is located near one of the boojums of the outer drop, as shown schematically in Fig. 1(g).

These shells are bipolar in nature; however, the inner droplet is not located at the center of the liquid crystal shell. To investigate the origin of the displacement of the inner drop from the center, we heat the liquid crystal above the nematic-to-isotropic transition temperature and bring the inner drop close to the center of the outer one. Upon cooling below the isotropic-to-nematic transition temperature, the inner drop initially remains in its original position; subsequently, however, it always moves towards one side. A typical example of this motion is shown in a series of images in Figs. 2(a)-2(c). The initial position is shown in bright field [Fig. 2(a)] while subsequent positions are shown in images obtained with crossed polarizers [Fig. 2(b) and 2(c)]. The droplet moves approximately along the direction set by the nearest pair of boojums until it reaches the outer surface, where the presence of the stabilizing polymer prevents coalescence with the continuous phase. To determine the nature of this motion, we measure the separation between the centers of the two drops, $r$, and find that it increases linearly in time as shown in Fig. 2(d). The resultant velocity is approximately $v=$ $0.7 \mu \mathrm{m} / \mathrm{s}$.
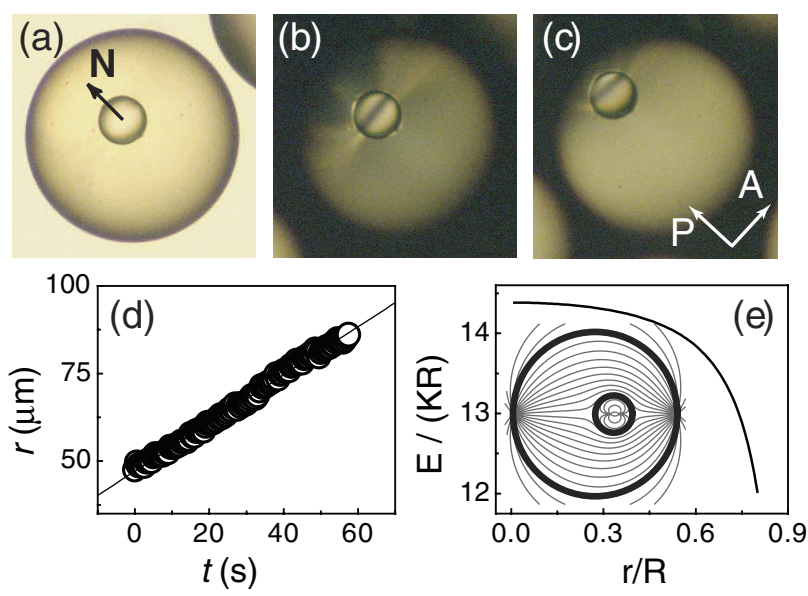

FIG. 2 (color online). Displacement of the inner drop driven by the elastic forces of the nematic liquid crystal: (a) $t=0 \mathrm{~s}$; (b) $t=13 \mathrm{~s} ;$ (c) $t=55 \mathrm{~s} . \quad 2 a=53 \mu \mathrm{m} . \quad 2 R=234 \mu \mathrm{m}$. (d) Within the experimentally observed range, the motion is uniform along the axis joining the two pair of boojums. (e) A trial texture for the nematic director in the experimental situation depicted in (a)-(c). The graph is a plot of the normalized energy, $E /(K R)$, vs $r / R$.

Our observations suggest that the elastic energy of the liquid crystal is minimized when the inner drop is located on the periphery of the larger one. To elucidate this behavior, we determine a trial texture for the director field that approximately matches the experimental texture and calculate the Frank free energy as the inner drop is displaced a distance $r$ from the center of the larger one. The trial texture is generated from an ansatz for the 2D texture, which is rotated about the line of motion of the inner drop to obtain the full 3D texture. To obtain the 2D ansatz, we use a conformal mapping technique that provides the flow lines of an incompressible fluid around a circular obstacle in a geometry that mimics our liquid crystal shell $[5,10]$. As an example, we show the 2D texture for the nematic director field when $r=a$ and plot the corresponding normalized energy, $E /(K R)$, with $K$ the elastic constant of the liquid crystal, as a function of a normalized centerto-center distance, $r / R$, in Fig. 2(e). The calculation shows that a droplet located exactly at the center of the larger drop is in unstable equilibrium. This corresponds to a director field with quadrupolar symmetry [11]. Any slight displacement of the inner droplet from the center decreases the separation of one pair of boojums, which corresponds to the charges of a dipole approaching one another; this decreases the energy. This results in an elastic force that drives the motion of the inner droplet. From the energy plot, we deduce that the elastic force increases with $r$. This suggests that the drop should accelerate as it moves towards the periphery of the larger one, whereas our experiment indicates that the speed is roughly constant. This discrepancy can be explained by the increased drag experienced by the drop as it approaches the boundary [12,13]; more detailed nematohydrodynamic calculations $[14,15]$ 
are required to fully explain the motion. Nevertheless, we can estimate the average elastic force on the droplet over its range of motion; it is of the order $K$. In our experiments, we can deduce an elastic force, $F_{e}$, from its balance with a rough estimate of the drag exerted by the liquid crystal over the inner droplet, $F_{v}=6 \pi \eta v a$; we obtain that $F_{e}$ is of order $K$, consistent with the theoretical estimation.

Although the boojum structure of the bipolar drop is observed for thicker shells, as the radius of the inner droplet is increased, the defect structure should ultimately transition to a qualitatively different configuration consisting of four defects arranged in the vertices of a tetrahedron $[6,7]$. We investigate this transition by generating double emulsions with thinner shells. The four-defect shell is expected when the shell thickness is below a critical value, $h^{*} \simeq e^{4} \sqrt{R} r_{c}$, where $r_{c}$ is the defect core radius [5]. When the shell thickness is greater than $h^{*}$, the shell with the boojum structure has the lowest energy. For $R=100 \mu \mathrm{m}$ and $r_{c}=10 \mathrm{~nm}$, we obtain $h^{*}=55 \mu \mathrm{m}$. Consistent with this estimate, we find only the boojum-based shell structure when $a \lesssim R / 2$. By contrast, when $a \gtrsim R / 2$, completely different behavior is observed. We are indeed able to observe the theoretically predicted four defects as indicated by the four dark spots on the surface of a typical thin shell of nematic liquid crystal, observed in bright field microscopy, shown in Fig. 3(a). The topological charge of each defect, as determined by the rotation of the director field around the defect core, is $+1 / 2$. This is deduced by observing the structure under crossed polarizers [Fig. 3(b)]; each defect has the expected pair of black brushes. These results are the first observation of the predicted defect structure for nematic order on a spherical surface [6,7]. Unexpectedly, however, the defects do not repel each other to occupy the four corners of a tetrahedron; instead they are all located at the top of the sphere. Moreover, for the thinnest shells we find a much richer scenario than anticipated theoretically.

In addition to the shells that contain four defects, we observe qualitatively different structures for similar values
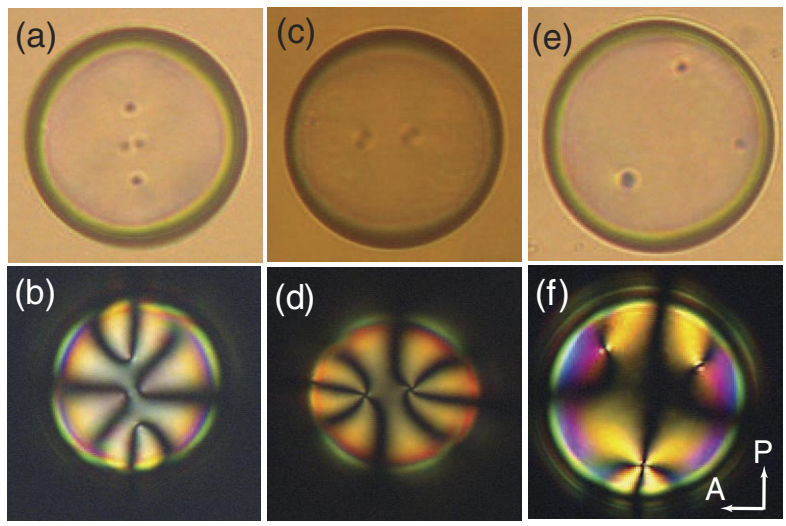

FIG. 3 (color online). Three type of thin shells commonly observed, distinguished by the number and type of defects: (a),(b) Four defects, (c),(d) two defects, and (e),(f) three defects. For all shells $2 a=103 \mu \mathrm{m}$ and $2 R=110 \mu \mathrm{m}$. of $a$ and $R$. We find shells with only two defects on the outer surface, as shown in Fig. 3(c). In this case, each defect has surface topological charge +1 as confirmed by the crosslike structure when observed through crossed polarizers, shown in Fig. 3(d). This two-defect structure is reminiscent of the boojum structure obtained for thicker shells. Even more strikingly, we routinely observe a threedefect structure, an intermediate state between the two other configurations. This remarkable hybrid configuration has three defects on the outer surface as shown by the bright field image in Fig. 3(e). It consists of two $+1 / 2$ and one +1 , as confirmed by rotating the drop under crossed polarizers, as shown in Fig. 3(f). Interestingly, we observe similar occurrences of each of the three different defect configurations; this suggests that each of the defect structures is equally accessible as the shells are made and that the energetic barriers between the various states are very high [5], preventing restructuring once the shells are formed.

Although forming double emulsions with very thin shells can produce three different defect configurations, we find that in each case, the defects are all located at the top of the drops. This results from the unavoidable
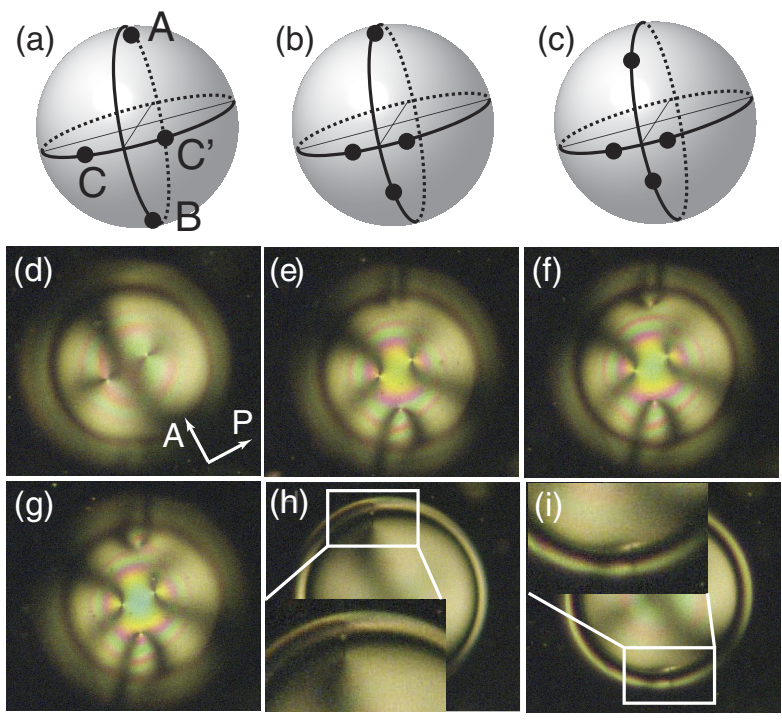

FIG. 4 (color online). Buoyancy driven motion of the inner drop in a nematic shell with four disclinations. $2 a=191 \mu \mathrm{m}$ and $2 R=204 \mu \mathrm{m}$. (a)-(c) are schematic visualizations of the defect motion. The inner drop moves along the direction of gravity, toward the midpoint of the arc connecting $C$ and $C^{\prime}$. The defects are first distributed throughout the shell and migrate in time to the top of the shell, ending in the final configuration shown in Fig. 3(a) and 3(b). (d)-(g) are cross-polarized images showing the time evolution of the defects motion: (d) $t=0$; (e) $t=70 \mathrm{~s}$; (f) $t=90 \mathrm{~s}$; (g) $t=110 \mathrm{~s}$. The plane of focus is always at the top of the double-emulsion drop. At approximately zero time, the other two-defect locations are shown in the crosspolarized images (h),(i). The particular defect configuration allows clear visualization of the disclination lines, as shown in the corresponding magnified images. 
nonzero thickness of the nematic shell and from the threedimensional character of the defects; the $+1 / 2$ defects are lines spanning the shell while the +1 boojums are halfradial and half-hyperbolic hedgehogs living in the outer and inner spherical surfaces, respectively. As a result, the defects overcome their mutual repulsion and migrate to the thinnest region of the shell where the energy associated with them is lowered. Since we systematically observe the defects at the top of the shells, this suggests that the thinning of the shell is not due to the nematic elasticity, which would generate shells with randomly oriented defect configurations. Rather, since the inner droplet is large, buoyancy forces are comparable to $K$, suggesting that buoyancy drives the motion of the inner droplet towards the top of the shells.

To test this hypothesis, we monitor the motion of the four defects in a baseball-like shell as the innermost drop is deflected away from the center and moves towards the top. We heat the sample above the isotropic-to-nematic transition temperature to remove any elastic forces on the inner droplet, gently adjust its position to be near the center of the outer drop, and then cool back into the nematic phase, where we observe the motion of the defects as the top of the shell thins [Figs. 4(a) $-4(\mathrm{~g})]$. Initially, when the inner droplet is near the center of the outer drop, the four defects are more widely separated although still not in the truly tetrahedral arrangement, as shown in Fig. 4(a) and 4(d). As the inner droplet moves upwards, the separation between each pair of defects decreases; this is more pronounced for the pair labeled $A$ and $B$, as shown schematically in Figs. 4(a)-4(c). Optical micrographs of these defects, visualized under cross polarizers, are shown in Figs. $4(\mathrm{~d})-4(\mathrm{~g})$. Defects $A$ and $B$ are initially nearly parallel to the image plane allowing us to directly visualize their structure; they are disclination lines extending through the shell, as can be seen in the magnified images in Figs. 4(h) and 4(i). As a result, their energy is lowered if they move to the thinnest portion of the shell. This overcomes the natural repulsion present in shells of uniform thickness, confirming our hypothesis. The time required for the droplet to move from its initial position to its final position is $t \sim 120 \mathrm{~s}$. To account for this, we balance the buoyancy force with the Stokes drag, using the lubrication approximation [12], $F_{v}=6 \pi \eta v \frac{a^{2}}{h}$, with $v=h / t$ the average velocity of the inner drop and $h=R-a$. We obtain $t=118 \mathrm{~s}$, which is consistent with our observations.

Our results highlight the fascinating range of structures that are driven by the interplay between topological constraints and the nematic order of liquid crystals. While the baseball-like structure originally predicted for thin nematic shells is indeed observed, it represents but one of a large number of other structures that arise from unavoidable thickness variations of the shells. Despite their complexity, the structures nevertheless fulfill the geometric constraints imposed by having the nematic confined between two spherical surfaces with tangential boundary conditions. Similar interesting defect structures can also be expected for shells with other crystalline order such as cholesteric or smectic.

This work was supported by NASA (Grant No. NAG32381), by the NSF (Grants No. DMR01-29804, No. DMR05-47230, and No. DMR-0602684), and by the Harvard MRSEC (No. DMR-0213805). A. F.-N. thanks the University of Almeria (leave of absence).

[1] P. S. Drzaic, Liquid Crystal Dispersions (World Scientific, Singapore, 1995).

[2] The term boojum was first introduced in: D. Mermin, Physica B+C (Amsterdam) 90, 1 (1977).

[3] P. Poulin et al., Science 275, 1770 (1997).

[4] H. Stark, Phys. Rep. 351, 387 (2001).

[5] V. Vitelli and D. R. Nelson, Phys. Rev. E, 74, 021711 (2006).

[6] T. C. Lubensky and J. Prost, J. Phys. II 2, 371 (1992).

[7] D. R. Nelson, Nano Lett. 2, 1125 (2002).

[8] A. S. Utada et al., Science 308, 537 (2005).

[9] H. Poincare, J. Math. Pures Appl. 1, 167 (1885); H. Hopf, Math. Ann. 96, 427 (1926). See also R. D. Kamien, Rev. Mod. Phys. 74, 953 (2002). The theorem establishes that the total topological charge of a vector or director field on a closed surface must equal the Euler characteristic of the surface, which is 2 for a spherical surface. Since each boojum has a charge of +1 , two boojums are required per spherical surface.

[10] The 2D director field corresponds to the flow lines of a source and a sink of an incompressible fluid located, respectively, at the two antipodal points on the outer drop, with a circular obstacle of relative radius $a / R$ located within the flow and displaced from the origin by $r / R$. In the limit of low $r / R$, the flow lines at a radial distance $r=R$ from the origin closely resemble a circle so that the boundary conditions on the outer drop are approximately satisfied. The conformal mapping that also enforces the correct boundary conditions on the inner droplet is $\Omega(v)=\log \left(\frac{v+1}{v-1}\right)+\log \left(\frac{a^{2}+(v-r)(1+r)}{a^{2}-(v-r)(1-r)}\right)$, where $v=z+i \rho ; \rho$ and $z$ are, respectively, the radial distance and the distance along the line of motion of the inner droplet, measured with respect to $r=0$. The flow lines are obtained by plotting the imaginary part of $\Omega(v)$.

[11] F. Brochard and P.-G. de Gennes, J. Phys. (France) 31, 691 (1970); S. Ramaswamy et al., Mol. Cryst. Liq. Cryst. 288, 175 (1996); T. C. Lubensky et al., Phys. Rev. E 57, 610 (1998).

[12] H. Brenner, Chem. Eng. Sci. 16, 242 (1961); J. Happel and H. Brenner, Low Reynolds Numbers Hydrodynamics (Prentice-Hall, Englewood Cliffs, NJ, 1965).

[13] L. Lobry and N. Ostrowsky, Phys. Rev. B 53, 12050 (1996).

[14] P. de Gennes and J. Prost, The Physics of Liquid Crystals (Oxford University Press, New York, 1995).

[15] A. D. Rey and M. M. Denn, Annu. Rev. Fluid Mech. 34, 233 (2002). 